\title{
Sport and Globalisation: Homogenization and Differentiation
}

\author{
Rui Gomez
}

Faculty of Sport Sciences and Physical Education,University of Coimbra, Portugal

The development of the worldwide market has motivated long-ranging consequences, not only at the level of growing economic interdependencies, but also in the globalization of cultures and lifestyles. At any of these dimensions, sport plays a role and contributes in its own particular way to globalization. Transnational organizations, worldwide events, transnational communities and transnational structures organised around the central theme of Sport provide good evidence of that phenomenon. However, the way these dimensions interrelate at a time of unorganised capitalism is based on disjuncture. Following this thesis, Appadurai (1996) has proposed an elementary scheme for the analysis of the disjuncture between the several dimensions of globalization, suggesting the notion of landscapes to underline the fluid and irregular shape of the capital flow, pertaining to both communications and lifestyles. By emphasising that globalization is intensively perceived according to, and influenced by the historical, linguistic and political contexts of the intervening players, the author deliberately focuses on the imagined worlds that help us construct those landscapes. In this paper, we will retrieve some of those theoretical leads and analyse three types of landscape in the leisure and sports contexts, in an attempt to demonstrate how their interrelation is one of disjuncture, where some dimensions promote sports homogenization while others push towards increasing differentiation. We will analyse the mediascapes (Sport as global spectacle), the technoscapes (the role of the new media and velocity in the creation of decontextualised global cognitive maps), and the ideoscapes (the role of images and the aesthetisation of the leisure sports experiences)

KEYWORDS sport, globalisation, localization, homogenization, differentiation, nature, culture, aesthetization

\section{The sports-gaze: the sport as a global spectacle}

According to postmodernists, we live in a society of the spectacle. Indeed, leisure and mass culture are truly fascinated with spectacles such as massive concerts or major sports events. The endless multiplication of international sports competitions dominates our current perception of the sports phenomenon and, to a certain extent, of the world itself. The Olympic Games, the World 
Championships of a number of different modalities, tennis and golf tournaments and the wide range of sports events which are brought into our homes on a daily basis are one of the most powerful magnets for crowd fascination. Due to its wide spread, sport has become one of the main engines powering globalization. By providing a temporary target for the collective consciousness, sports events ensure an illusion of instantaneity as well as one of self-referencing. The universalisation of lifestyles warranted by the sports-vision is closely followed by maximised individualisation within the private sphere. This is hardly a recent phenomenon; however, the unprecedented development of homogenization and coding of sports rules all around the planet, as well as the fact that the sports world has been now taken over by strong financial motivations, has largely strengthened this correlation over the last two decades: the sports-vision of the world as the key metaphor for globalization.

Live TV broadcasting, allowing near real-time electronic transmission of the sports events, has led to a new planet-scale spatialisation, which, in turn, is ruled by time and by a unified consciousness of events. Following the pace of competitions and the sports calendars, the mass media host new offers which do more than just broadcasting or influencing events; instead, these new offers have grown to be true creators of reality. Iconomania has turned champions and high-level sports competitors into advertising models and public figures with whom youngsters are keen to identify; the lifestyles, the image and the body of the new icons have become desirable models of social success. At the dawn of the new century, sport is the best known entertainment industry and the one most viewed around the globe.

However, such an upward movement of the viewed sport failed to be followed by a similar trend in actual physical and sports activity. This is a particular trait of the more general gap between the society of the spectacle and the reduced body experience. This ambience of non-action has brought back the discussion on the role of the gaze in the organization of leisure activities to the foreground. Although the high-level sports performance follows a logic that is utterly different from that ruling the body fitness activity, the sportivisation of society as promoted by the sports gaze has dictated leisure behaviours in line with rituals of self-discipline, rationalisation and regularity. It is a fact that sport as a discipline has been recycled in the leisure context, under the form of spontaneous body freely obeying individual rhythms. The argument for psy-activities based on a kind of ritual of the self has gained ground. Nevertheless, as stressed by Andrieu (2004, p. 55) «the paradox of our society lies in displaying the unequal competition typical of the sport as spectacle and, at the same time, developing the individual cult of the body. The individual accepts to himself the search for a natural harmony with his own body, while on a collective basis - and it has been increasingly facilitated by mediators each one of us submits to the imposing ideology of artificially professionalised bodies».

In order to understanding this paradox, we must go back to the very genesis of the sportivisation of society. The rationalisation, the quantification and the continuous striving for recordbreaking are the three characteristics that turn sport into a social phenomenon similar to the grassroots of industrial society and modernisation. Within the sports context, sports competition and the corresponding measurement of performance arise as the technologies providing the grounds for the art of rationalizing the sports gesture and simultaneously establishing the social images which make calculable people acceptable and necessary (Hacking 1986). The measurement of sports performance expresses an entirely new power of body rationalisation. Firstly, because it turns the sportsman into a rational, measurable, calculable and settable field; secondly, because it makes it possible to build a written archive of a sportsman's individual performance, and to establish his relative position within a given population; thirdly, because it sets the standard and defines deviations. The consolidation of such technology has put the competitive, leisure and health sports activity under a regime of recording, 
evaluation and measurement, and the society has been reorganised under a regime of productivity and performance. The images constructed by the sports activity include an ethical system applicable to the leisure activities. In fact, what is seen today as a soft way of listening to and following one's body (jogging, for example) is frequently associated with pain, dieting, compliance with certain prohibitions and typical lifestyles that lead the sportsman to embrace asceticism, though it is perceived as hedonism.

That is precisely the paradox of the sports-gaze. A new power-knowledge complex has formed with the possibility of establishing objective measures of the human value. The sportsman's mark works both as the registering of a past performance and as the quantified expression of the human value. When aggregated, marks become a competence measurement underlying the subject, arising as a new equation of the self against the others. For quite a long time, individual behaviours have been hidden under the veil of discretion. Knowing each one's value, by learning his/her life story, used to be the exclusive feature of a restricted social group which actively disclosed personal achievements. In any case, life narratives were devised to build a monument memory. The sports performance is not intended to build a future memory; instead, it is a feat for possible and always transitory use. The memorable man is replaced with the calculable man, subject to rationality and to the principle of merit. Thanks to the statistical tools normally associated with the techniques of noting, recording and archiving sports marks, it is possible to estimate each individual's value, and to evaluate and characterise the different groups at the same time. On the one hand, a new modality of power is born, in which one is assigned the status corresponding to what seems to be his/her intrinsic value; and on the other hand, individual differences become evident in the whole population. In a single move, competitive sport initiates the construction of an apparently universal structure of opportunities, standardised forms of merit and strategies to obtaining status and achieving social mobility.

Sport is a social construction and, as such, it represents the dominant ideas and values. Highcompetition sport has been used in the United States to promote the interpretations intended to demonstrate how social life should work (Coakley 1998). Record-breaking presents sports competitions as one of the dominant metaphors for the society's democratic-meritocratic design (Bromberger 1995). Values such as work, team spirit, ambition, unification, effort, modesty, respect and discipline, among others, leverage the belief in the effectiveness of self-overcoming. If a distinctive belief presides today to leisure physical activities, that is precisely the restless search for the intensity of the self, though standardization and rigidity may have been replaced with spontaneity and flexibility, two concepts that are far better reputed in our days. In any case, living intensively and at the pace required by the ephemeral nature of both events and products is one golden rule of the consumer society. Let us move forward, then, to discuss the dimension of velocity and its impact on sports globalisation.

\section{Velocity as a metaphor for globalisation}

The story of the sports globalisation can be told in a variety of ways. One way of telling this story is through the body experience involving physical and sports activities in which players engage as tourists; it is a known fact that a wide tourism market has developed associated with sports, whether it be the sports spectacle attracting crowds of tourists to great and medium-sized sports events of a global or local nature, or the fitness sports, attracting an increasing number of tourists seeking across borders events in golf, surfing, wind-surfing, skiing, skateboarding, sailing, cycling, running, hiking, climbing, hunting, fishing, etc. 
The central issue to address is to understand how and by which means the individual subject, isolated from a psychological point of view, relates with the objective - total and yet socially scattered - reality of the places visited. The base concept used to reflect on this contradiction between terms will be the notion of cognitive map. Cognitive maps are a sort of imaginary solution found for real contradictions, responding to the need to cognitively unify a necessarily more fragmented daily reality.

We believe this is an interesting concept for understanding one of the basic paradoxes of the entire tourist behaviour, including the sports tourist behaviour: on the one side, the change of locations and the movement implied by the touristic activity make us believe in unlimited physical freedom. On the other side, the physical experience on which this new distant-near geography was built, the experience of velocity, has turned space into a mere function of movement.

Partly, this is an effect of velocity and globalisation. Nowadays, we travel at a speed inconceivable to our ancestors. Motion and travelling related technologies allowed human settling to shift farther away from the crowded centres towards the peripheral areas. Therefore, space has turned into a means whose purpose is movement itself. We have an increasing tendency to classify urban spaces depending on accessibility. As the urban space converts into a function of movement, it also becomes less stimulating. The driver in the car, the pilot in the plane or the passenger in the highspeed train only desires to cut across space and has no intention of having his attention caught by the surrounding environment.

Increased life speed has triggered a number of effects. The most obvious one concerns the amplification of our consciousness of simultaneity. Simultaneity as a consciousness is dissociated from the time-space relations: spatially remote processes now come together in our immediate involvement.

As we are increasingly bombarded with information about what we should do and what we should be, we grow increasingly aware of the cost of opportunity. The cost of opportunity is defined as the individual balance of the multiple opportunities lost in the pursuit of another one. All the choices we make today in our leisure and travelling time entail what is referred to by Virilio (1991) as the small death, because we have the feeling that we never know whether the choices we ruled out might have been more fulfilling or rewarding than the ones we opted for. Considering that the quality and the amount of options seem to expand endlessly, we tend to experience stronger dissatisfaction and have the feeling that we are always missing something.

From the individual point of view, such dissatisfaction produces a greater tendency to fragmentation, discontinuity and division. This reinforces the notion of hyperreality, once the distinction between the real thing and its imitation, between sign and significance, is simply eliminated. The consumer is presented with ever more comprehensive simulation technologies: holiday resorts that mimic and amplify the look and feel of the exotic; closed spaces in cold regions that mimic luxuriant sites in tropical beaches; exotic areas displaying references recognisable by the tourists from other continents; enclosed spaces amplified by means of simulated images allowing us to play golf at the hotel, to play football in our own room, or to ride a bike in wild landscapes without leaving the convenient resort facilities.

In this state of permanent simulation, what is geographically distant strikes one as terribly familiar and what is geographically near creates an illusion of strangeness. Tele-presence or teletravelling present us with a reality that is strangely near and similar to our own, creating what Guattari categorizes as generalized nomadism, in which all experiences are mutually equivalent, however much one travels around the world. 


\section{The return to nature as a metaphor for localisation}

The touristic experience of travelling is also an experience of disconnection from the surrounding space, as velocity prevents one from paying due attention to the natural and human landscape, acting as a duplicated experience of sensorial deprivation. This ambience of hyperreality has been the setting for a return to nature, visible in the growing demand for outdoor leisure activities and the trend registered in many sports to acquire a more ecological dimension. Eco-tourism, ecoleisure and eco-sports have seen their numbers of participants grow, also as a reaction to the artificiality of life, velocity and urban sedentarism. The wild nature arises as the public's preferred reference, while the bucolic or rural nature is relegated to second place. This imaginary construction of a wild nature, a sort of lost paradise, has been largely determined by the new search for local roots. Outdoor sports practices play a role in this search for an identity able to develop new roots in a given territory and a given place. The surfer's quest for the perfect wave, the paraglider's search for the most suitable place for sweeping flights, or the hiker's ambition to find a secret and exotic walking track is part of a symbolism simultaneously based on wandering and contemplative quietness. The outstanding aspect of that search is the invention of new paths and new significances for the routes drawn in natural places intended for sports leisure.

These images are the so-called cognitive maps. All the concepts we use to represent the reality have a spatial, a physical and a symbolic thickness, within which the real can be identified. However, cognitive maps constructed this way fail to be a true copy of the physical reality and are instead allegories intended to tell a story over a story. According to Jameson's theory (1984), cognitive maps must be used as a form of constructing a previously non-existing unity. The author argues that the cartographic production of space owes a debt to the different periods in the history of capitalism, and that a very specific development has been accomplished in each one of those periods. Likewise, this lead may be followed to characterise present day leisure activities.

The period of unorganised capitalism we live in today is marked by the dismantling of the great colonial empires and the exponential growth of the world market. The massive presence of multinationals has weakened the regulatory capacity of national economies. National states have progressively lost their ability to continuously regulate the sphere of production, and this has prompted profound changes in the production's spatial configuration, as well as in regional configuration. The extensive market expansion unfolds in parallel with the intensive expansion of new technologies allowing borders to be instantaneously overcome. As a consequence, the postmodern space involves an unrivalled suppression of distance and a saturation of space, with an inevitable crisis occurring at the level of its representation.

According to Jameson, the dispersion and the fragmentation of the subjects' living conditions has made it increasingly difficult for them to attain global comprehension. The expansion of capital has operated the separation between the world experience and our representation of the world. In other words, the excess and speed of local experiences has enlarged the deficit in understanding the world. The daily experience is becoming more and more limited to a small fraction of the world, the image of a landscape or a monument captured by mobile phone or camera, even though the substance of that experience no longer coincides with the place in which it actually takes place. The truthfulness of the limited experience of a block in Lisbon, London or Barcelona may happen in India, the USA or Jamaica. At this stage of unorganised capitalism, leisure is not differentiated from the other spaces, according to well defined and regulated territories. Instead, it is a floating space where bodies circulate at high speed, with no predefined routes and often immaterially, like in cyberspace.

Surfing a wave or surfing the net, modifying the body sensations in a sensorial isolation room or in a virtual game, are different ways of incorporating the world in each one of us. The body is 
perceived as a flow of information and sensations with no particular context and with no identity unity. Once nothing is fixed or stable, nothing can be apprehended through experience. This is the context in which new cognitive maps are sought and constructed in their relation with the natural space. Cognitive maps, as previously seen, depend on a change of locations. We are well aware of how the practice of urban leisure activities almost always requires a place or equipment and, for most of the time, a change of locations inside or around the urban centre. These mobilities are not always rational and may be determined by other encounters, by the need to share a common leisure space or the drive to leave the more immediate urban surroundings and reach out for nature. Regardless of being totally wild or somehow man-altered nature, these contexts favour the construction of new cognitive maps related with nature sports activities.

\section{The visual culture and the aesthetization of life}

Cities show a trend to develop a cosmopolitan lifestyle where poor loyalty to localities and a strong sense of flexibility stand out among a number of other characteristics. This has compelled urban citizens to constantly look for new experiences, sensations and relationships. Therefore, urban lifestyles are burdened with functional and psychological overload, time-related pressure and slow daily mobility, a range of factors that may seriously compromise the quality of life; social relations fall hostage to acceleration and impersonality.

The very design of a city follows the ongoing trend to harmonise leisure, consumption and culture. These elements are unified by design for a certain period. What is really taking place in the urban space is a progressive transition from the economic, functional and production-centred axis to a cultural and aesthetic axis, focused on consumption and leisure activities. This shift is the key issue underlying the concepts of consumption or postmodernity cultures. The triumphant entry of the audiovisual in the list of cultural practices is one of the most striking aspects of this phenomenon.

The urban culture can hardly be accused of limiting cultural practices to the restricted codes of elitist culture - another effect of globalisation. The blend of elitist, mass and folk contents and formulae has profoundly changed the legitimising and validating entities, and has reconfigured the monopoly of those who can make «true» speeches on symbolic and cultural goods. This is the context in which cultural mediators find an entirely renewed role. A variety of professional backgrounds becomes compatible under the privileged relationship these communication agents maintain with culture, by being the holders of assets such as information, reflexivity and critical distance. Cultural mediators lend an analytical meaning to the concept of lifestyle, which expresses the individuals' ability to construct and manage ways of living based on self-expression and stylistic self-conscience. The fact that life is being turned into a work of art, and that lifestyles and everyday social relationships have such a strong built-in plasticity, becomes fairly understandable when we consider the growing aesthetisation and stylisation of life, as individuals in general and some social groups in particular, namely the younger ones, tend to make increasingly bigger investments of time and money in composing a certain style.

The urban space is a competitive arena for the post-industrial city. The growing expansion of services, trade, and tertiary and quaternary sectors, drives the reshaping of the economic, urbanistic and social structure of the cities, particularly in city centres. In such a city, the spotlight is on economic activities linked with symbolic production, and the professional activities of the symbolic analysts related with culture industries, mass-media, advertising, education, design, marketing, architecture, decoration and leisure - including sports leisure - take the lead. 
This new sports leisure market features mobility, fluidity and a blend of consumers motivated by the wish to socialise as much as by the intention to engage in a specific sports activity. They are often transitional and nomadic urban places appropriated by certain groups, even when they have not been especially designed as sports leisure venues. Generally, those spaces combine three characteristics typical of a new visual culture powered by the search for a lifestyle:

- places where the staging, the symbolism and the space signature are vital and appeal directly to the consumer;

- places of socialising, where animation intermingles with the intimate relationship, combining exotic materials, sounds and postmodern functions typical of zapping and fast consumption;

- places offering a number of different areas with multiple roles: resting areas, discussion areas, show areas, meeting areas, services areas, etc;

In a setting where everyone approaches consumption as a way to create a style of his/her own, the value underlined by Nike's slogan - Just do it - becomes the main guideline in selecting the sports leisure practices. This principle applies equally to consumption and sports practices. Just do it means that consumption takes place in the free play of sensations and desires, and the moment when that choice is made becomes one and the same with the self-fulfilling moment of the buying. Sports practices too are now ruled by this aesthetic principle overrating the expression of each one's individuality or immediate pleasure as a form of constructing a life project. The trademarks, the sneakers, the place, the rarity or the exotic nature of one's activities, and the tribe one wants to belong to, are more important criteria than pursuing a record-breaking mark, an enhanced performance or even victory.

The basic principles of competitive sports no longer fit the new production and consumption regime based rather on the use of the credit card. Society as we once knew it, organised on the assumptions of hard-working, life-long employment, self-disciplined use of time and deferred satisfaction, is definitely gone. It has been replaced with the consumer society where the notions of temporary, transitory and ephemeral are present in all the objects and all the consumption experiences.

The competitive sports based on the ethical principles of hard-work struggle to overcome a deeply set crisis, caused by their incapacity to attract and recruit the younger populations. The notion of training based on an ethics of duty and a rigid approach to pleasure and entertainment seems completely obsolete in the face of the new juvenile values. Youngsters approach sports in much the same way as they would buy a pair of sneakers and do nothing more than giving it a try before moving on (just do it). Because of the growing penetration of individualism and consumerism, the classic collective organisation of sport has faced countless obstacles in its effort to diversify and adapt. At the same time, new trends have arisen, especially those organised around sports tourism and leisure. Getting an immediate reward and living an intense experience through consumption is a value that is also present in the sports leisure. And it is interesting to see that, while some of these trends seem to induce a simultaneous effect of homogenization - the globalisation of Californian activities, for instance - others build around the search for the exotic and local particular traits - the tradition of local games is a vivid example.

Five main trends can be identified:

- health tourism and leisure, associated with active practices and the new hygienism;

- sports and cultural tourism and leisure, patent in the return to traditional games, cultural heritage and local or regional physical culture; 
- eco-tourism and eco-leisure activities as a way of enjoying or preserving nature, landscape and natural surroundings;

- risk tourism and leisure, through extreme or challenging activities often associated with counterculture phenomena and creative expressions such as graffiti, hip-hop and rag;

- entertainment tourism and leisure, both for spectacle or self-expression purposes, associated with animation and mixed events of mass culture and alternative body uses.

This is the new map of urban and outdoor leisure activities, both of them based on informality and profiting from the difficulties experienced by traditional sports in attracting new sportsmen. Ever since the boom of Californian sliding sports, such as skateboarding, inline skating and others, the imaginary notion of free time has been deeply reshaped and even the oldest outdoor activities including jogging, climbing, canoeing or sailing - have endured a number of changes and originated new myths. Adventure, challenge, incitement, vertigo, different appropriation of spaces, signs of belonging such as clothes, materials or footwear - these are all values containing a certain dramatic intensity that is closely related with media-covered adventurous and challenging achievements. Much faster than sports professionals, architects and designers have perceived the new trends and have been translating that sense of challenge, speed, sliding and itinerancy required by the new informal practices into the new urban equipment and natural spaces.

The challenge cultural mediators are faced with today is that of being capable of successfully adapting to the new times and keeping track of the globalisation trends emerging, without neglecting the sports and leisure opportunities offered by the senior institutions. Those trends reveal one of the most stimulating aspects of the globalisation processes: while they trigger a simultaneity and simulation effect where everyone seems to be doing the same thing, they also generate local alternatives and resistances based on individualisation and on typically postmodern reflexivity, allowing everyone to think they are doing things their own way.

\section{REFERENCES}

Andrieu, B. (2004). A nova Filosofia do corpo. Lisboa: Instituto Piaget.

Appadurai, A. (1996). Modernity at Large. Cultural Dimensions of Globalization. University of Minnesota.

Bromberger et alli (1995). Le Match de football: Ethnologie d'une passion partisane à Marseille, Naples et Turin. Paris: Maison des Sciences de l'Homme.

Chazaud, P. (2004). Management du tourisme et des loisirs sportifs de pleine nature. Lyon: PUS.

Coakley, J.J. (1998). Sport in Society (6 ${ }^{\mathrm{a}}$ Ed.). Boston: McGraw -Hill.

Eco, U. (1986). Faith in Fakes. London: Secker \& Warburg.

Hacking, I. (1986). Making up people. In T. C. Heller, M. Sosna e D. E. Wellberg (Eds.), Reconstructing Individualism (pp. 222-236). Stanford: Stanford University Press.

Huyssen, A. (1986). After the great divide. Bloomingtone Indianapolis: Indiana University Press.

Jameson, F. (1984, 1991). Postmodernism or the Cultural Logic of Late Capitalism. Durham, NC: Duke University Press.

Kerckhove, D. (1997). A Pele da Cultura. Lisboa: Relógio d'Água.

Roseneau, J. (1990). Turbulence in World Politics. Brighton: Harvester.

Virilio, P. (1991). The Aesthetics of Disappearance. New York: Semiotext(e). 